\title{
High-resolution peripheral quantitative CT in rheumatology
}

Cheryl Barnabe, Stephanie Finzel, Kathryn S. Stok and Piet Geusens

We read with interest the Perspectives article by Filippucci et al. (Progress in imaging in rheumatology. Nat. Rev. Rheumatol. 10, 628-634; 2014). ${ }^{1}$ This article is a very informative concise paper on MRI and musculoskeletal ultrasonography. However, we were surprised that no mention was made of high-resolution peripheral quantitative CT (HR-pQCT), previously highlighted in a Review in this journal, ${ }^{2}$ and we would like to call attention to its emerging potential in the field of advanced diagnostic imaging.

HR-pQCT was initially developed to study bone diseases such as osteoporosis, and has contributed to a better understanding of the separate behavior of trabecular and cortical bone structures and the different cortical envelopes during ageing, in metabolic bone diseases and during fracture healing; ${ }^{2}$ moreover, it allows for calculation of bone strength in vivo using finite-element analysis. ${ }^{3}$ Due to its ability to evaluate bone in $3 \mathrm{D}$ at a high resolution $(82 \mu \mathrm{m})$ and with minimal irradiation ( $5 \mu \mathrm{Sv}$ to image a $0.9 \mathrm{~cm}$ region of interest), HR-pQCT is being intensely investigated in the evaluation of bone architecture in the small joints of the hands in rheumatoid arthritis, ${ }^{4-6}$ psoriatic arthritis, ${ }^{7}$ and hand osteoarthritis. ${ }^{8}$ To what degree can this method now be rated as "progress in imaging" in rheumatology?

Firstly, an increased number of, and earlier stages of, erosions are visible with HR-pQCT than on plain radiography, as evidenced by the work of Stach et al. ${ }^{4}$ Secondly, quantification of erosions in terms of size (width, depth and volume) together with changes in trabecular bone is possible, and has been studied in crosssectional and longitudinal studies ${ }^{5,9-11}$ and in comparison with MRI. ${ }^{6}$ Thirdly, a $3 \mathrm{D}$ assessment of joint space is described using quantitative analysis algorithms. ${ }^{12,13}$ Fourthly, 3D measurement of bone microarchitecture in rheumatoid arthritis patients showed altered results with disease activity. ${ }^{14}$ None of these $3 \mathrm{D}$ parameters can currently be evaluated by other imaging techniques at such high resolution.

In conclusion, HR-pQCT is, besides MRI and musculoskeletal ultrasonography, also promising "progress in imaging" for clinical research in rheumatic disease of the hand joints. SPECTRA (Study Group for $\mathrm{X}$-treme CT in Rheumatoid Arthritis) ${ }^{15}$ is an international collaboration between rheumatologists, radiologists, biomedical engineers, physicists and clinical researchers in bone metabolism and osteoimmunology that has provided fertile ground for crossdisciplinary research, united by the desire to certify the validity of the research findings from the application of this technology to the assessment of inflammatory arthritis.

University of Calgary, Department of Medicine, 3330 Hospital Drive NW, Calgary, AB T2N 4N1, Canada (C.B.). Department of Internal

Medicine 3, University of Erlangen-Nuremberg, Erlangen 91054, Germany (S.F.). Institute for Biomechanics, ETH Zurich, Wolfgang-PauliStrasse 14, 8093 Zurich, Switzerland (K.S.S.). Department of Internal Medicine, Subdivision of Rheumatology, CAPHRI/NUTRIM, Maastricht University Medical Centre, P. Debyelaan 25, Postbus 5800, 6202 AZ, Maastricht, Netherlands (P.G.).

Correspondence to: $C . B$. ccbarnab@ucalgary.ca

\section{Acknowledgements}

This correspondence was written by the authors on behalf of the Study Group for X-treme Computed Tomography in Rheumatoid Arthritis (SPECTRA).

\section{Competing interests}

The authors declare no competing interests.

1. Filippucci, E., Di Geso, L. \& Grassi, W. Progress in imaging in rheumatology. Nat. Rev. Rheumatol. 10, 628-634 (2014).

2. Geusens, P. et al. High-resolution in vivo imaging of bone and joints: a window to microarchitecture. Nat. Rev. Rheumatol. 10, 304-313 (2014).

3. Cheung, A. M. et al. High-resolution peripheral quantitative computed tomography for the assessment of bone strength and structure: a review by the Canadian Bone Strength Working Group. Curr. Osteoporos. Rep. 11, 136-146 (2013).
4. Stach, C. M. et al. Periarticular bone structure in rheumatoid arthritis patients and healthy individuals assessed by high-resolution computed tomography. Arthritis Rheum. 62 . 330-339 (2010).

5. Fouque-Aubert, A. et al. Assessment of hand bone loss in rheumatoid arthritis by highresolution peripheral quantitative CT. Ann. Rheum. Dis. 69, 1671-1676 (2010).

6. Srikhum, W. et al. Quantitative and semiquantitative bone erosion assessment on high-resolution peripheral quantitative computed tomography in rheumatoid arthritis. J. Rheumatol. 40, 408-416 (2013).

7. Finzel, S. et al. Bone anabolic changes progress in psoriatic arthritis patients despite treatment with methotrexate or tumour necrosis factor inhibitors. Ann. Rheum. Dis. 72 1176-1181 (2013).

8. Finzel, S. et al. Inflammatory bone spur formation in psoriatic arthritis is different from bone spur formation in hand osteoarthritis. Arthritis Rheumatol. 66, 2968-2975 (2014).

9. Finzel, S. et al. Interleukin-6 receptor blockade induces limited repair of bone erosions in rheumatoid arthritis: a micro CT study. Ann. Rheum. Dis. 72, 396-400 (2013).

10. Finzel, S. et al. Repair of bone erosions in rheumatoid arthritis treated with tumour necrosis factor inhibitors is based on bone apposition at the base of the erosion. Ann. Rheum. Dis. 70, 1587-1593 (2011).

11. Topfer, D., Finzel, S., Museyko, O., Schett, G. \& Engelke, K. Segmentation and quantification of bone erosions in high-resolution peripheral quantitative computed tomography datasets of the metacarpophalangeal joints of patients with rheumatoid arthritis. Rheumatology (Oxford) 53, 65-71 (2014).

12. Barnabe, C. et al. Reproducible metacarpal joint space width measurements using $3 \mathrm{D}$ analysis of images acquired with highresolution peripheral quantitative computed tomography. Med. Eng. Phys. 35, 1540-1544 (2013).

13. Burghardt, A. J. et al. Quantitative in vivo HR-pQCT imaging of 3D wrist and metacarpophalangeal joint space width in rheumatoid arthritis. Ann. Biomed. Eng. 41 2553-2564 (2013).

14. Zhu, T. Y. et al. Alterations of bone density, microstructure, and strength of the dista radius in male patients with rheumatoid arthritis: a case-control study with HR-pQCT. J. Bone Miner. Res. 29, 2118-2129 (2014).

15. Barnabe, C. \& Feehan, L. High-resolution peripheral quantitative computed tomography imaging protocol for metacarpophalangeal joints in inflammatory arthritis: the SPECTRA collaboration. J. Rheumatol. 39, 1494-1495 (2012). 